\title{
Centromere identity from the DNA point of view
}

\author{
Miroslav Plohl • Nevenka Meštrović • Brankica Mravinac
}

Received: 27 November 2013 / Revised: 28 March 2014 / Accepted: 1 April 2014 /Published online: 25 April 2014

(C) The Author(s) 2014. This article is published with open access at Springerlink.com

\begin{abstract}
The centromere is a chromosomal locus responsible for the faithful segregation of genetic material during cell division. It has become evident that centromeres can be established literally on any DNA sequence, and the possible synergy between DNA sequences and the most prominent centromere identifiers, protein components, and epigenetic marks remains uncertain. However, some evolutionary preferences seem to exist, and long-term established centromeres are frequently formed on long arrays of satellite DNAs and/or transposable elements. Recent progress in understanding functional centromere sequences is based largely on the high-resolution DNA mapping of sequences that interact with the centromere-specific histone $\mathrm{H} 3$ variant, the most reliable marker of active centromeres. In addition, sequence assembly and mapping of large repetitive centromeric regions, as well as comparative genome analyses offer insight into their complex organization and evolution. The rapidly advancing field of transcription in centromere regions highlights the functional importance of centromeric transcripts. Here, we comprehensively review the current state of knowledge on the composition and functionality of DNA sequences underlying active centromeres and discuss their contribution to the functioning of different centromere types in higher eukaryotes.
\end{abstract}

Keywords Centromere · Satellite DNA · Transposable elements · Transcription

\section{Introduction}

An essential function of genetic material in any living organism is its faithful segregation, the role which is in eukaryotes

M. Plohl $(\bowtie) \cdot$ N. Meštrović $\cdot$ B. Mravinac

Division of Molecular Biology, Ruđer Bošković Institute, Bijenička

54, 10002 Zagreb, Croatia

e-mail: plohl@irb.hr determined by the centromere. The centromere includes the core or functional centromere domain, a specialized locus at which microtubules attach to the complex multiprotein structure of the kinetochore in order to segregate chromosomes in mitosis and meiosis. The core centromere domain is surrounded by large blocks of pericentromeric heterochromatin (also called the pericentromere), primary sites of sister chromatid cohesion. Centromere functionality is vital for all eukaryotic organisms. In addition to understanding its role as a biological structure, studying the centromere is also highly relevant from a biomedical point of view, because abnormalities in centromeric function are often lethal or associated with various congenital and acquired diseases, such as cancer, infertility, and birth disorders (reviewed in Thompson et al. 2010).

Centromeres are considered to be shaped by both genomic and epigenetic mechanisms, but the synergy between DNA sequences, protein components, and epigenetic marks is still not well understood. In the absence of a universal DNA sequence, species-specific histone $\mathrm{H} 3$ variant CENH3 (CENP-A in mammals, CID in Drosophila melanogaster, Cse4 in Saccharomyces cerevisiae) is the most prominent protein identifier of centromere function. Related forms of this protein have been detected in all studied active centromeres of single-cell and multicellular eukaryotes (Black and Bassett 2008; Malik and Henikoff 2009). CENH3 replaces the canonical histone $H 3$ in such a way that arrays of CENH3-based nucleosomes alternate with those containing canonical H3 (Blower et al. 2002; Sullivan and Karpen 2004). In humans and flies, canonical $\mathrm{H} 3$ is in turn epigenetically modified in the centromere, by dimethylation at lysine 4 (H3K4me2), and thus distinctive from the histone $\mathrm{H} 3$ in adjacent pericentromeric heterochromatin, which is marked by methylation at lysine 9 (H3K9me). These differences qualify centromeric chromatin as a unique chromatin type centrochromatin (Sullivan and Karpen 2004). 
In the budding yeast $S$. cerevisiae, centromere function depends on a short, about $100 \mathrm{bp}$ long DNA sequence motif. These centromeres are referred to as simple or point centromeres (Hyman and Sorger 1995). In all other eukaryotes, centromeres are founded on repetitive DNA arrays of several hundred kilobase, commonly known as complex or regional centromeres (Pluta et al. 1995). A single centromere is normally formed on each chromosome in a locus which is on the cytogenetical level recognized as a primary constriction of the monocentric chromosome. However, there are exceptions, and some organisms have holocentric chromosomes that lack a primary constriction and comprise of a centromere dispersed in many subdomains along the entire chromosome length (Dernburg 2001).

Mostly, due to limitations in sequencing and assembly of long arrays of nearly-identical repeats, our knowledge on the long-range functional organization of centromeric DNA is rather limited, and centromeres still represent the last frontiers in genome assemblies and sequence annotations (Hayden and Willard 2012). Here, we review the rapidly progressing field of functional centromere genomics. We present data relating DNA sequences and their functional interactions in different centromere types of higher eukaryotes, and point to the significance of transcriptional potential of centromeric sequences.

\section{Repetitive DNA sequences are the most common centromere components}

Two classes of highly abundant repetitive sequences, satellite DNAs (satDNAs) and transposable elements (TEs), represent major DNA components of many centromeric regions. Both groups of sequences are extremely divergent, and understanding the mechanisms of their accumulation, diversification, protein-binding capacity, and linear distribution is essential for a complete picture of centromere genomics, both from a structural and functional perspective. Characteristics of functional DNA sequences and other abundant DNAs contributing to centromere region of the most common model organisms of higher eukaryotes are presented in Table 1.

SatDNAs are a class of diverse tandemly repeated DNA sequences that comprise long arrays localized in a tightly packed heterochromatin. Features of satDNA sequences in centromeric regions have already been reviewed in detail (Plohl et al. 2008, 2012). A recent comprehensive bioinformatic analysis of centromeric satDNAs in a number of animal and plant species confirmed the rapid evolution of DNA sequences in these areas (Melters et al. 2013). Despite the extreme diversity of satDNA sequences, some sequence segments can be shared among heterologous repeats. The best known example is the conserved 17 bp long sequence motif, the CENP-B box, which is specific for alpha-satDNA in humans (Ohzeki et al. 2002), as well as in various subclasses of alphoid repeats in mammalian species (Alkan et al. 2011). This motif is a binding site for the protein CENP-B, which probably facilitates kinetochore formation (Masumoto et al. 2004), but might also play a role in rearrangements of satDNA sequences (Kipling and Warburton 1997). The presence of CENP-B box-like motifs in unrelated satDNAs of some distant invertebrates and plants suggests its potential functional relevance in non-mammalian organisms (Mravinac et al. 2005; Canapa et al. 2000; Meštrović et al. 2013; Gindullis et al. 2001).

SatDNAs evolve according to the principles of concerted evolution. Within the genome, mutations are homogenized among repeats of the satDNA by the mechanisms of nonreciprocal sequence transfer, such as unequal crossover, gene conversion, rolling circle replication, and transposition-related mechanisms (Dover 1986). Although the centromere was traditionally treated as a region of suppressed recombination, unequal crossing-over and gene conversion have been identified as the most widespread mechanism involved in satDNA dynamics (Mahtani and Willard 1998; Smith 1976; Talbert and Henikoff 2010). Nevertheless, recent studies on primates and plants postulated mechanism of segmental duplication as an important evolutionary force in the massive amplifications of satDNA arrays and long range rearrangements of (peri)centromere regions (Horvath et al. 2005; Ma and Jackson 2006). At the population level, satDNAs become fixed as a result of random assortment of genetic material in meiosis. As species diverge, satDNAs accumulate changes as a consequence of mutations and turnover mechanisms in separate lineages generating species-specific satDNA arrays (Dover 1986). However, rapidly accumulating differences in species-specific satDNA profiles can also be accomplished by amplifications/contractions of repeats existing in a so-called library of satDNAs common to related genomes. The hypothesis was originally proposed by Fry and Salser (1977) and experimentally proved by Meštrović et al. (1998). As predicted by the theory of concerted evolution, a small bias in favor of homogenization of a particular set of repeat variants would lead to extreme conservation of satDNAs (Ohta and Dover 1984; Strachan et al. 1985), observed in various organisms, for example, in sturgeons (De la Herran et al. 2001) and beetles (Mravinac et al. 2002). Because of the above mentioned specificities, the scenario of satDNA evolution unifies array homogeneity and long-term sequence stability together with the ability of the satDNA library to act as a reservoir of sequences that allow rapid changes through expansions and contractions of arrays (Plohl et al. 2008).

Nevertheless, it is difficult to understand the rapid evolution of satDNAs in a centromere solely by sequence dynamics of tandem repeats, especially in the light of the centromere structure-function paradox (Eichler 1999). The phenomenon of rapid evolution of centromeric DNA and protein 
Table 1 Centromere DNA features in higher eukaryote model organisms

\begin{tabular}{|c|c|c|c|c|}
\hline $\begin{array}{l}\text { Centromere type } \\
\text { according to functional } \\
\text { DNA sequence }\end{array}$ & Species/common name & $\begin{array}{l}\text { Characteristics of functional } \\
\text { DNA sequence(s) }\end{array}$ & $\begin{array}{l}\text { Other abundant DNAs } \\
\text { contributing to centromere region }\end{array}$ & References \\
\hline \multirow[t]{5}{*}{ Satellite DNA } & Homo sapiens/human & $\begin{array}{l}\text { alpha-satDNA [171 bp] in all } \\
\text { centromeres; chromosome- } \\
\text { specific subfamilies; higher- } \\
\text { order organization }\end{array}$ & $\begin{array}{l}\text { Monomeric forms of alpha- } \\
\text { satDNA, diverse non-alphoid } \\
\text { satDNAs (gamma, beta, Sat I, } \\
\text { II, III) and LINE elements in } \\
\text { pericentromeric regions }\end{array}$ & $\begin{array}{l}\text { Willard and Waye 1987; } \\
\text { Waye and Willard 1989; } \\
\text { Rudd and Willard 2004; } \\
\text { Rudd et al. 2006; } \\
\text { Sullivan et al. 2011; } \\
\text { Schueler et al. 2001 }\end{array}$ \\
\hline & $\begin{array}{l}\text { Drosophila } \\
\text { melanogaster/fruit } \\
\text { fly }\end{array}$ & $\begin{array}{l}\text { Dp1187 centromere: AATAT } \\
\text { and AAGAG satDNAs }\end{array}$ & $\begin{array}{l}\text { Dp1187 centromere: LTR } \\
\text { retrotransposons (HMS } \\
\text { Beagle, 412, and Bel), non- } \\
\text { LTR (LINE-like) retroposon } \\
(F) \text { and 359-bp satDNA }\end{array}$ & Sun et al. 1997, 2003 \\
\hline & $\begin{array}{l}\text { Mus musculus/house } \\
\text { mouse }\end{array}$ & $\begin{array}{l}\text { Minor satDNA }[120 \mathrm{bp}] \text { : } \\
\text { homogenous family in all } \\
\text { centromeres }\end{array}$ & $\begin{array}{l}\text { satDNAs: MS3 [150 bp] in } \\
\text { centromeric core, Major } \\
\text { satDNA [234 bp] and MS4 } \\
{[300 \mathrm{bp}] \text { in pericentromeric }} \\
\text { regions }\end{array}$ & $\begin{array}{l}\text { Guenatri et al. 2004; } \\
\text { Kuznetsova et al. } 2006\end{array}$ \\
\hline & $\begin{array}{l}\text { Arabidopsis thalianal } \\
\text { thale cress }\end{array}$ & $\begin{array}{l}\text { pAL1 satDNA [180 bp]: } \\
\text { homogenous family in all } \\
\text { centromeres }\end{array}$ & $\begin{array}{l}\text { LTR-retrotransposon (Athila) in } \\
\text { centromere core, multiple } \\
\text { families of LTR } \\
\text { retrotransposons and 5SrRNA } \\
\text { in pericentromeric regions }\end{array}$ & $\begin{array}{l}\text { Nagaki et al. 2003; } \\
\text { Kumekawa et al. } 2000\end{array}$ \\
\hline & Pisum sativum/pea & $\begin{array}{l}13 \text { distinct satDNAs families } \\
{[50-2,094 \mathrm{bp}] \text { localized in }} \\
\text { various combinations in } \\
\text { different centromeres }\end{array}$ & $\begin{array}{l}\text { satDNAs: TR2, TR3, and TR5 in } \\
\text { pericentromeric regions }\end{array}$ & Neumann et al. 2012 \\
\hline \multirow[t]{2}{*}{$\begin{array}{l}\text { Satellite DNAs and } \\
\text { retrotransposons }\end{array}$} & Oryza sativa/rice & $\begin{array}{l}\text { CentO satDNA }[155 \mathrm{bp}] \text { and } \\
\text { CRR retrotransposon in all } \\
\text { centromeres }\end{array}$ & $\begin{array}{l}\text { Different retrotransposon } \\
\text { families belonging to Ty3/ } \\
\text { gypsy-class }\end{array}$ & $\begin{array}{l}\text { Dong et al. 1998; Cheng } \\
\text { et al. } 2002\end{array}$ \\
\hline & Zea mays/maize & $\begin{array}{l}\text { Retrotransposons CRM1 and } \\
\text { CRM2 and CenC satDNA } \\
{[156 \text { bp }] \text { in all centromeres }}\end{array}$ & $\begin{array}{l}\text { Retrotransposons CRM3 and } \\
\text { CRM4 }\end{array}$ & $\begin{array}{l}\text { Zhong et al. 2002; } \\
\text { Wolfgruber et al. } 2009\end{array}$ \\
\hline Retrotransposons & Triticum spp./wheat & $\begin{array}{l}\text { CRW, Quinta and Weg } \\
\text { retrotransposon families }\end{array}$ & $\begin{array}{l}\text { Different CRW retrotransposons } \\
\text { families (Ty3/gypsy-class) }\end{array}$ & Li et al. 2013 \\
\hline \multirow[t]{3}{*}{ Repeats and non-repeats } & Equus caballus/horse & $\begin{array}{l}\text { satDNAs: different ECA families } \\
\text { [221-475 bp], 37cen } \\
{[221 \mathrm{bp}], \text { and } 2 \mathrm{PI}[23 \mathrm{bp}]} \\
\text { localized in various } \\
\text { combinations; repeat-free ch11 } \\
\text { centromere }\end{array}$ & NA & $\begin{array}{l}\text { Piras et al. 2010; Alkan } \\
\quad \text { et al. } 2011\end{array}$ \\
\hline & Gallus gallus/chicken & $\begin{array}{l}\text { Chromosome specific satDNAs } \\
{[1.8-3.2 \mathrm{~kb}] \text { in centromeres of }} \\
\text { eight macrochromosomes, } \\
\text { CNM satDNA [ } 42 \mathrm{bp} \text { ] in } \\
\text { some microchromosomes and } \\
\text { in ch6 and ch9; repeat-free ch5, } \\
\text { ch27, chZ centromeres }\end{array}$ & NA & Shang et al. 2010 \\
\hline & $\begin{array}{l}\text { Solanum tuberosum/ } \\
\text { potato }\end{array}$ & $\begin{array}{l}\text { Six chromosome specific } \\
\text { satDNAs }[979 \text { bp to } 5.4 \mathrm{~kb}] \\
\text { repeat-free ch } 4, \text { ch6, ch } 10 \\
\text { ch } 11, \text { and ch1 } 2 \text { centromeres }\end{array}$ & NA & Gong et al. 2012 \\
\hline
\end{tabular}

components in spite of conserved centromere function has been referred to as the centromere paradox (Henikoff et al. 2001). In this regard, evolution of CENH3 is subject to positive selection in Drosophila (Malik and Henikoff 2001) and Arabidopsis (Talbert et al. 2002), and probably in general (Talbert et al. 2004) because of its interactions with changing
DNA components. Centromeres are thus not defined only by epigenetic factors but also through interactions between repetitive DNA and protein components, mediated by meiotic drive (Dawe and Henikoff 2006). In other words, rapid evolution of centromere satDNA sequences is possible only assuming coevolution with CENH3 and other DNA-binding proteins. 
Because satDNAs are the major DNA components of heterochromatin, differences in their composition can be linked with reproductive isolation and speciation (Bachmann et al. 1989). Differences among individuals in the centromere region accumulate as a consequence of centromere drive, leading to reduced compatibility of homologous chromosomes in hybrids and ultimately to postzygotic isolation, thus triggering speciation (Henikoff et al. 2001). The role of satDNA in reproductive isolation caused by rapid centromere evolution has been recently studied in detail in monkeyflowers (Fishman and Saunders 2008) and Drosophila (Ferree and Barbash 2009).

Another repetitive component of importance for centromeric regions are transposable elements (TEs), DNA sequences which can move to new genomic locations and form interspersed repeats if replicated in the process of movement (Kazazian 2004; Tollis and Boissinot 2012). According to the mechanisms of transposition, TEs are categorized as RNAmediated (retroelements such as long terminal repeat (LTR) and non-LTR-retrotransposons) or DNA-mediated (DNA transposons). In addition to sequence segments coding for their own enzymes and thus being self-sufficient in the process of mobility, enzymes of autonomous elements can trail a large number of various non-autonomous copies.

Among TEs, LTR-retrotransposons in particular accumulate frequently in centromeres and pericentromeres of both plants and animals (e.g., Pimpinelli et al. 1995; Copenhaver et al. 1999; Schueler et al. 2001; Cheng et al. 2002). TEs belonging to the chromovirus clade of Ty3/gypsy LTRretrotransposons are widely distributed in centromeres of angiosperms. It has been proposed that they are targeted to centromeres by a specific motif located at the C-terminus of their integrase (Neumann et al. 2011). Molecular determinants that need to be recognized by this motif in order to trigger specific integration are probably sequence-independent heterochromatin marks, although their exact nature has not yet been unambiguously identified (Neumann et al. 2011; Tsukahara et al. 2012). In addition to active transposition, centromere-specific retrotransposons can become significantly enriched in centromeric regions as a consequence of multiple rounds of segmental duplication, a process which can also be responsible for massive amplifications of satDNA arrays (Ma and Jackson 2006).

Despite differences in the structure, organization, dynamics, and mechanisms of spread, a growing number of reports link TEs and satDNAs. A whole unit or a segment of a TE can be amplified in tandem, although the direction of transition between the two types of repetitive sequences is not always clear (Macas et al. 2009). For example, a part of the mammalian retrotransposon L1 shares similarity with a segment of the satDNA repeat in whales (Kapitonov et al. 1998). Internal tandem repeats of nonautonomous miniature inverted repeat transposable element
(MITE) from the cupped oyster Crassostrea virginica resemble satDNAs in several other mollusks (Gaffney et al. 2003). In plants, a hypervariable region of one LTRretrotransposon was found expanded into tandem repeats of a satDNA in the pea (Pisum sativum) genome (Macas et al. 2009). Similarly, Zea mays centromeres became enriched in tandem repeats derived from LTRs and untranslated regions of two unrelated centromere-specific retrotransposons, what probably happened in two independent evolutionary events (Sharma et al. 2013).

\section{Repeat-based centromeres}

The majority of eukaryotes studied in terms of centromeric DNA have monocentric chromosomes with large regional centromeres. Functional centromeric domains of these chromosomes are usually inserted into blocks of pericentromeric heterochromatin, a compartment composed of Mb-sized arrays of satDNAs. Arrays are in general much longer than necessary for centromeric function. For instance, functional centromere domains in Drosophila comprise only of 15$40 \mathrm{~kb}$, which is comparable to the minimum length of 30 $70 \mathrm{~kb}$ of alpha-satDNA in a functional centromere of human artificial chromosomes (Okamoto et al. 2007).

Details on the complexity of organizational patterns and contribution of particular sequence types to repeat-based centromeres differ significantly among species (Fig. 1). For example, global sequence characterization of rice centromeric satDNA CentO by next generation high-throughput sequencing and ChIP experiments with CENH3 could not reveal any particular differences between monomers included in the functional centromere and pericentromeric arrays (Macas et al. 2010). A comparable uniform distribution of nearlyidentical repeats of species-specific highly-abundant satDNAs (up to $50 \%$ of the genome) in centromeric and pericentromeric heterochromatin of all chromosomes can be anticipated in some beetle species of the order Coleoptera (Palomeque and Lorite 2008). It has been proposed that the lack of chromosome-specific satDNA variants (Fig. 1a) indicates high efficiency of sequence homogenization in the bouquet stage of meiotic prophase, in which all chromosomes of the complement align together (Durajlija Žinić et al. 2000; Mravinac and Plohl 2010). In contrast, well-known examples of satDNAs localizing to pericentromeric and centromeric regions are the mouse major and minor satDNA, respectively, (Guenatri et al. 2004; Kuznetsova et al. 2006).

The distribution of centromeric satDNAs can also be chromosome specific (Fig. 1b). The best studied example is the complex organizational pattern of centromeric sequences in human chromosomes. Two basic types of alpha-satDNA, monomeric and higher-order repeat (HOR), characterize human centromeric regions (Willard and Waye 1987; Rudd and 
Fig. 1 Schematic presentation of functional DNA sequences in different centromere types
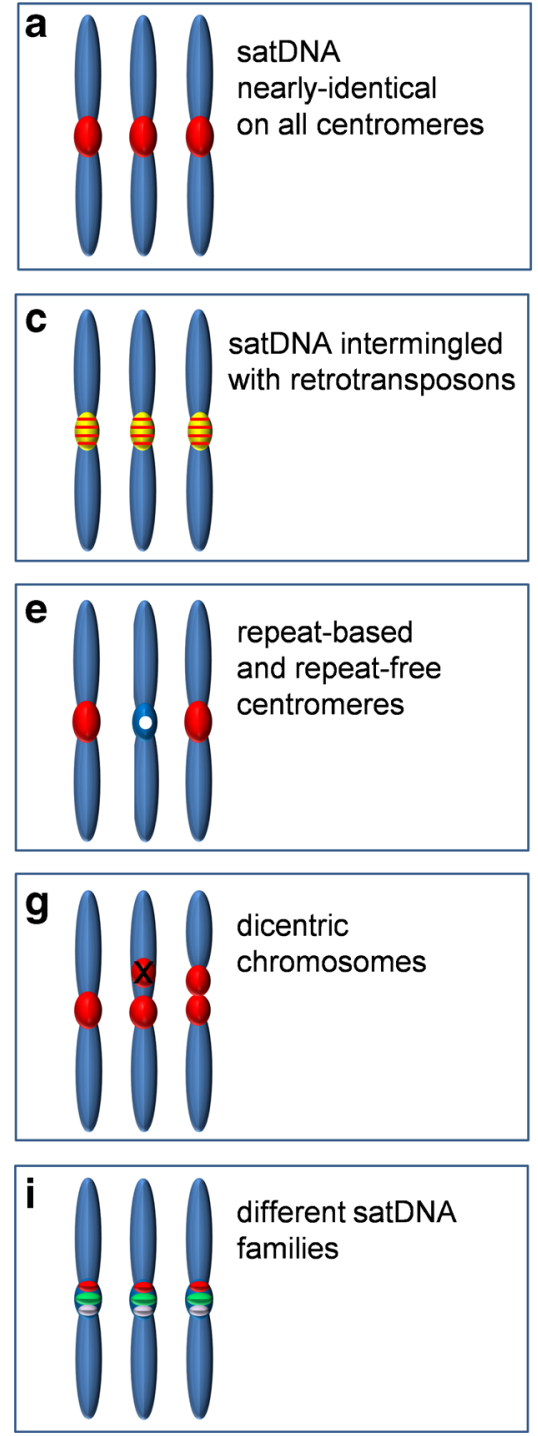
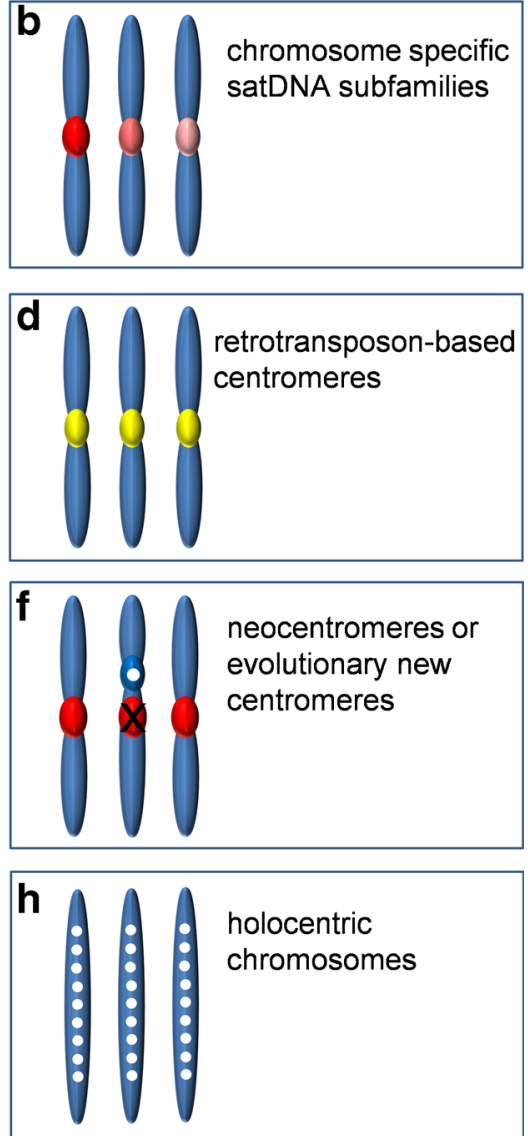

repetitive sequences

$\circ$ non-repetitive sequences

centromere inactivation
Willard 2004). All regular human centromeres are formed on tandemly repeated HOR units composed of 2 to over 20 diverged 171-bp-long monomers, and HORs are usually chromosome specific (Rudd et al. 2006). However, only a fraction of HOR arrays of human alpha-satDNA underlies active centromeres, while the rest, flanked by monomeric repeats, contributes to pericentromeric heterochromatin (Spence et al. 2002; Lam et al. 2006; Mravinac et al. 2009; Sullivan et al. 2011). Comparably, in the domestic dog, CENP-A chromatin immunoprecipitation (ChIP) experiments suggested monomer sequence subtypes of two related satDNAs as functional centromere sequences (Hayden and Willard 2012). Recent efforts combining genomic and ChIP-obtained data on human alphasatDNA allowed the possibility for comprehensive functional mapping of centromeric areas and led to a model in which the centromere is defined by sequence features and contextdependent epigenetic interactions (Hayden et al. 2013).
The diversity of DNA sequences localized in functional centromeres and/or pericentromeres has been evidenced not only in terms of different satDNAs and their organizational forms, but also in terms of other sequences' contribution. Different interspersion patterns of tandemly repeated DNA and TEs are found in many species (Fig. 1c). The centromeric fraction of human HORs is mostly devoid of inserted TEs or other sequences, while pericentromeres are frequently interrupted by unrelated satDNAs (e.g., gamma-satellite and SatIII) and LINE elements (Schueler et al. 2001). Different plants such as maize, rice, and wheat turned out to be valuable models for studying the specificities of centromere DNA sequence organization, particularly because of the presence of substantial portions of centromere-specific retrotransposons. Retrotransposons are extensively intermingled with satDNAs and both sequence types mark functional parts of some plant centromeres (Ma 
et al. 2007). For instance, functional rice centromeres are characterized by CentO satDNA and the centromere-specific retrotransposon CRR (Cheng et al. 2002). A recent study in the wild rice Oryza brachyantha showed that CentO satDNA repeats as well as CRR retrotransposons have completely disappeared and are replaced by a new functional centromeric CentF satDNA in a short evolutionary time (Lee et al. 2005).

Detailed mapping of the repeat content and arrays of complete centromeres in some chromosomes of maize (Wolfgruber et al. 2009) and wheat (Li et al. 2013) revealed species-specific centromeric retrotransposons as predominant CENH3-associated DNA sequences (Fig. 1d). Maize centromeres still contain small amounts of CentC satDNAs, detected as functional centromeric sequences in other maize inbreds (Kato et al. 2004; Wolfgruber et al. 2009) and related to the CentO satDNA in rice (Cheng et al. 2002). Similar replacements of functional centromeric satDNA with retrotransposons occurred in wheat, followed by consecutive introduction of new functional retrotransposons. All these replacements occurred in a very short evolutionary time, $<0.5 \mathrm{MY}$ (Li et al. 2013). In principle, older retrotransposons typically lie outside of the functional centromere (Wolfgruber et al. 2009; Li et al. 2013) and can be compared with the distribution of LINE and other TEs in pericentromeres of human chromosomes (Schueler et al. 2001). It has been hypothesized that retrotransposons may accumulate in active centromeres because of favored integration into an epigenetically modified centromere environment, and not because of preferred association with CENH3 nucleosomes (Lamb et al. 2007; Wolfgruber et al. 2009).

Complex organization of centromeric regions is further supported by the presence of protein coding genes or gene candidates in centromeric chromatin of D. melanogaster (Smith et al. 2007), rice (Wu et al. 2004; Nagaki et al. 2004), and wheat (Li et al. 2013), although the insertions of this type were not observed in Arabidopsis (Hosouchi et al. 2002) and human (Schueler et al. 2001).

\section{Organisms with both repeat-based and repeat-free centromeres}

From the methodological standpoint, due to the abundance of satellite repeats in eukaryotic species, it is understandable that the literature to date mostly describes the cases of centromeric regions rich in repetitive sequences. However, the development of chromatin immunoprecipitation and usage of CENH3 variants as the most reliable markers of active centromeres enabled high-resolution DNA mapping of interacting sequences. Consequently, there are an increasing number of reports documenting the organisms that possess both repeatbased and repeat-free centromeres (Fig. 1e). Horse Equus caballus centromeres are enriched for satellite sequences but the functional centromere of chromosome 11 lacks any tandem repeats (Piras et al. 2010). The extended cytogenetic analysis of congeneric species revealed that donkey and two zebra species contain several pairs of chromosomes with satellite-less centromeres (Piras et al. 2010). The chicken genome with 10 pairs of macrochromosomes, 28 pairs of microchromosomes, and $\mathrm{Z} / \mathrm{W}$ sex chromosomes represents the first avian karyotype with molecular cytogenetic characterization of each chromosome (Masabanda et al. 2004), and thus has been a powerful resource for studying the genetic makeup. Thorough identification of centromeric DNA showed that the majority of chicken centromeres are founded on chromosome-specific satDNA spanning several hundred kilobase of homogeneous repetitive arrays, while centromeres of chromosomes 5, 27, and Z, spanning only $\sim 30 \mathrm{~kb}$, are devoid of tandem repeats (Shang et al. 2010). The presence of the two distinct types of centromeres has also been evidenced in plants. In the potato, Solanum tuberosum, no satellite repeats were discovered in centromeres of five pairs of chromosomes, whereas six potato centromeres harbor megabase-sized chromosome-specific satellite repeat arrays (Gong et al. 2012). Similar to chicken, centromeric satellites in potato share partial sequence similarity to different retrotransposon sequences (Gong et al. 2012).

\section{Neocentromeres and evolutionary new centromeres (ENCs)}

Neocentromeres are fully functional centromeres that arise at ectopic DNA loci not previously associated with kinetochore proteins (Fig. 1f). In humans, the majority of neocentromeres evidenced in clinical phenotypes rescue acentric chromosome fragments in cells with severe chromosomal rearrangements (Marshall et al. 2008). As the neocentromeres described to date show notable divergence of underlying DNA sequences and chromosome positions, the sequence attributes that might be favorable to their formation have not yet been established. Most of them are located in gene-poor regions with no apparent association with heterochromatin (Alonso et al. 2010), and although some of them form on repetitive DNA (Hasson et al. 2011), none of them are associated with alpha-satellite DNA. In addition to human cells, neocentromere formation and function have also been studied in different model organisms such as D. melanogaster, Schizosaccharomyces pombe, Candida albicans, and several plant species (reviewed in Burrack and Berman 2012).

Evolutionary new centromeres (ENCs), also known as repositioned centromeres, are centromeres that moved to a new position along a single chromosome without any observable chromosomal rearrangements or phenotypic consequences. Once repositioned, ENCs are transmitted through generations and become fixed in the population. Since they 
can be identified exclusively by comparing the ancestral and derived position of a specific centromere, systematic karyotype analyses of related organisms are crucial. So far, the best studied model group is primates and it has been proved that nine macaque chromosomes possess ENCs (Ventura et al. 2007), whilst six human centromeres are evolutionarily new (reviewed in Rocchi et al. 2012). ENCs have also been revealed in other mammals (e.g., Carbone et al. 2006; Rocchi et al. 2012), birds (Kasai et al. 2003), and plants (Han et al. 2009). Although they arise in anonymous sequences, ENCs gradually incorporate repetitive arrays. In macaque, all the nine ENCs over time accumulated large arrays of alphasatDNA becoming indistinguishable from other macaque centromeres. At the same time, the inactivated centromeres completely lost their satellite arrays (Ventura et al. 2007). Similarly, centromere repositioning in cucurbit species was accompanied by the gain of centromeric satDNA repeats in ENCs and the loss of pericentromeric heterochromatin in inactivated centromeres (Han et al. 2009).

What can be learned from neocentromere and ENC phenomena is that a centromere potentially can be seeded in any unique sequence, albeit the repetitive DNA setup provides a preferred chromatin environment for centromere maintenance. The hypothesis that repeat-free centromeres represent a primordial form is in accordance with the occurrence of neocentromeres and their maturation into repeat-based centromeres by the accumulation of satellites and retrotransposons (Kalitsis and Choo 2012).

\section{Dicentric chromosomes}

Each chromosome normally possesses a single centromere, though genome rearrangements can generate chromosomes with two centromeres (Fig. 1g). In general, dicentric chromosomes are inherently very unstable because of anaphase bridge formation resulting in broken or rearranged chromosomes. Nevertheless, in some cases, dicentric chromosomes are stabilized due to inactivation of one of the two centromeres, which allows the structural dicentric to act as a functional monocentric during cell divisions. The exact mechanism of centromere inactivation has not been completely elucidated; however, studies of naturally occurring and engineered dicentrics in different organisms predominantly indicate epigenetic changes. In the fission yeast, $S$. pombe, $99 \%$ of the cells harboring an artificial dicentric chromosome died, but in $70 \%$ of the survivors, one of the centromeres was functionally silenced by the loss of Cnp1 (the yeast CENH3 homolog), depletion of euchromatic histone modifications $\mathrm{H} 3 \mathrm{~K} 9 \mathrm{ac}$ and H3K14ac, and by becoming enriched for the heterochromatic $\mathrm{H} 3 \mathrm{~K} 9 \mathrm{me} 2$ mark without associated alterations in the DNA sequence (Sato et al. 2012). Epigenetic centromere inactivation has also been documented in maize dicentric $\mathrm{B}$ chromosomes. Without changing the sequence of underlying DNA, one of the B chromosome centromeres becomes nonfunctional by histone CENH3 depletion (Han et al. 2006) and increasing methylation of the underlying DNA (Koo et al. 2011). A structural tricentric chromosome in wheat acts like a functional monocentric by keeping active the large centromere, while at the same time both of the small centromeres, enriched for heterochromatic histone modifications $\mathrm{H} 3 \mathrm{~K} 27 \mathrm{me} 2$ and $\mathrm{H} 3 \mathrm{~K} 27 \mathrm{me}$, are inactivated (Zhang et al. 2010). Dicentric chromosomes in humans can be quite stable, and it has been known for two decades that some human dicentric chromosomes also stay functional dicentrics through multiple cell divisions (Sullivan and Willard 1998). Stimpson et al. (2010) recently showed that the human dicentrics, being functionally monocentric, undergo centromere inactivation through different processes: (1) by epigenetic mechanisms or (2) by size reduction of the alpha-satDNA array associated with CENP-A. Human chromosome HSA17, characterized by the two alpha-satellite arrays D17Z1 and D17Z1-B, is an example of a regular human chromosome structurally arranged as a dicentric that behaves as a functional monocentric. Its functional centromere is predominantly linked to the D17Z1 array (Maloney et al. 2012). However, in vitro and in vivo studies proved that the HSA17 functional centromere can also assemble at D17Z1-B, and its location is inherited through multigenerational families. The structural differences in the D17Z1 and D17Z1-B HOR arrays imply genomic factors that, together with epigenetic mechanisms, influence centromere specification in humans (Maloney et al. 2012). In other words, the analyses of natural and engineered dicentric chromosomes indicate that epigenetic plasticity, but also subtle genetic features of centromerecompetent DNA sequences, plays an important role in defining centromere identity.

\section{Holocentric centromeres}

In contrast to monocentric, holocentric chromosomes have a long kinetochore plate with spindle fibers attached along the entire chromosome length (Dernburg 2001) (Fig. 1h). Based on cytological studies, it has been shown that holocentric chromosomes are scattered among plant and animal kingdoms arising at least 13 independent times during evolution (Mola and Papeschi 2006). A more precise understanding of centromeric function in holocentric species, based on immunodetection of CENH3 homologs, has been intensively analyzed only in the nematode, Caenorhabditis elegans, and a few other species. In spite of polyphyletic origin, immunodetection of the corresponding $\mathrm{CENH} 3$ proteins in mitotic chromosomes of C. elegans (Buchwitz et al. 1999) and the plant Luzula (Nagaki et al. 2005; Heckmann et al. 2011) shows common structural features in the form of dispersed 
CENH3 distribution during interphase and prophase. In both species, diffuse centromeres are distributed along each chromatid except in the telomeric regions (Heckmann et al. 2011). Data on the DNA sequences underlying holocentric centromeres are generally lacking. Nevertheless, a recent study of animal and plant species shows that the genomic content of tandem repeats in holocentric species differs greatly (Melters et al. 2013). The C. elegans genome contains only a few tandem repeats (Hillier et al. 2007). ChIP analysis shows that even $\sim 50 \%$ of this genome is associated with CENH3, but association loci are not correlated with repeat density (Gassmann et al. 2012). In contrast, comprehensive characterization of holocentric Luzula elegans shows that $61 \%$ of its genome is built of highly repetitive DNAs, including over 30 highly divergent satellite families, while $33 \%$ of the genome comprises Ty1/copia LTR retrotransposons of the Angela clade (Heckmann et al. 2013). Although retrotransposons in L. elegans are uniformly distributed along the chromosomes, they are not centromere-associated. Similarly, different satDNAs are present as blocks preferentially accumulated on chromosome ends which are declared as non-centromeric regions. However, a portion of centromere domains in the related holocentric species Luzula nivea is composed of scattered clusters of satellite LCS1 which display significant similarity to the major centromeric satellite of monocentric chromosomes of some Oryza species (Haizel et al. 2005). These data suggest that satDNA can be an important centromere determinant in this holocentric species. In support of this, a study of novel meta-polycentric chromosomes in the pea $P$. sativum, which represents the first example of an intermediate between monocentric and holocentric centromeres, demonstrates that all functional centromere domains in the pea are tightly associated with clusters of 13 distinct satDNA families and with one family of retrotransposons (Neumann et al. 2012). The pea centromeres have from three to five explicit CENH3-containing regions composed of different families of satDNAs (Fig. 1i).

\section{Transcription of centromeric sequences}

The non-coding nature of repetitive sequences in centromeres and pericentromeres led to the opinion that centromeres are transcriptionally inactive. However, new evidences show that small-interfering RNAs (siRNAs) transcribed from pericentromeric tandem repeats in $S$. pombe modify the heterochromatin. In brief, transcription of pericentromeric sequences in the form of double stranded RNAs and their processing into siRNAs by the ribonuclease Dicer proved to be crucial in heterochromatin assembly and transcriptional silencing (Volpe et al. 2002). Impairment of the RNA interference (RNAi) pathway resulted in severe chromosome segregation defects in S. pombe (Hall et al. 2003). Subsequent studies on higher eukaryotic species showed a link between the RNAi machinery and heterochromatin-mediated transcriptional silencing in plants (Zilberman et al. 2003), flies (Drosophila; Pal-Bhadra et al. 2004), worms (C. elegans; Grishok et al. 2000), and mammals (Fukagawa et al. 2004). However, the ultimate impact of RNAi on heterochromatin assembly and chromosome segregation is less straightforward suggesting different mechanisms of the RNAi pathway in complex genomes (Chan and Wong 2012). In hybrid chicken cells carrying a human chromosome, loss of Dicer led to defects in centromere heterochromatin and chromosome segregation, pointing out the importance of siRNA for heterochromatin assembly (Fukagawa et al. 2004). Similarly to chicken cells, Dicer deficiency in mouse embryonic stem (ES) cells caused accumulation of pericentric satellite transcripts, but there are still controversies related to the impact of the RNAi machinery on mammalian centromere assembly (Kanellopoulou et al. 2005; Murchison et al. 2005). Kanellopoulou et al. (2005) reported loss of DNA methylation and of histone $\mathrm{H} 3$ modification $\mathrm{H} 3 \mathrm{~K} 9 \mathrm{me} 3$ at the pericentromeric regions of Dicer-deficient ES cells and suggested that Dicer participates in the maintenance of centromeric heterochromatin structure. In contrast, Murchison et al. (2005) concluded that the RNAi pathway is not essential for the regulation of heterochromatin assembly in mouse ES cells because in their experimental system Dicer loss had no significant effect on cytosine methylation nor changed $\mathrm{H} 3 \mathrm{~K} 9 \mathrm{me} 3$ status at the centromere. More recent work on $S$. pombe suggests that the observed defects may be indirectly related to exosome RNA machinery (a multiprotein complex capable of degrading various RNA types), which acts in parallel with RNAi and promotes heterochromatin formation (Reyes-Turcu et al. 2011).

In addition, a great progress has also been made in determining non-siRNAs transcripts in the centromere of higher eukaryotes. The data suggest transcriptional competence of the entire centromere (both the centromere core and the pericentromere) and heterogenous transcripts appear to be variable in size and structure (Gent and Dawe 2012). They can be transcribed from both strands or display strand-specific characteristics (Topp et al. 2004; May et al. 2005). Some of them are exclusively nuclear while the other form cytoplasmatic polyadenylated RNA (Vourc'h and Biamonti 2011). Increasingly, evidence suggests an impact of centromeric transcripts on development, cell differentiation, and response to environmental stimuli.

Pericentromeric major satDNA in mice is highly transcribed during embryogenesis, and transcripts are responsible for reorganization of pericentromeric satDNA into chromocenters. Disruption of these transcripts led to developmental arrest indicating their role in de novo heterochromatin formation and proper developmental progression (Probst et al. 2010). In humans, polyadenylated RNA transcripts from the 
pericentromeric region of the $\mathrm{Y}$ chromosome are involved in trans-splicing in the CDC2L2 kinase mRNA generating a testis-specific isoform (Jehan et al. 2007). This example illustrates specific regulation of euchromatic gene expression by pericentromeric transcripts and provides a link between satDNA transcription and cell differentiation. The overexpression of centromeric RNA transcripts may be the result of derepression of heterochromatic regions under disease or stress conditions. So, it has been proposed that the differential transcription of human pericentromeric satellite III in response to heat-shock stress might be a consequence of inhibition or saturation of the RNAi machinery in the pericentromeric region (Jolly et al. 2004). BRCA1-deficient tumor cells show defective pericentromeric heterochromatin formation which leads to the disruption of gene silencing and activation of the pericentromeric alpha-satDNA transcription (Zhu et al. 2011). Derepression of satDNA transcription has also been detected in many human epithelial tumors, but it is not clear whether satDNA transcription causes or is a consequence of genomic instability and tumorigenesis (Ting et al. 2011).

In addition to the analysis of pericentromeric regions, an ever-growing number of studies on the centromere core domain demonstrates the transcription of repetitive sequences from this region and suggests a contribution of these transcripts to centromere/kinetochore assembly and maintenance (Gent and Dawe 2012). The single-stranded centromeric alpha-satellite RNA and the centromere protein CENP-C associate and facilitate nucleoprotein assembly (CENP-C, innercentromere protein INCENP, and INCENP-interacting protein survivin) at the human mitotic centromere (Wong et al. 2007). Inhibition of RNA polymerase II activity, which results in depletion of alpha-satellite RNA in mitotic human cells, reduces CENP-C binding at the kinetochore and leads to chromosome missegregation (Chan et al. 2012). Similarly, Minor satDNA transcripts from the mouse centromere are integral components of the CENP-A chromatin fraction and associate with proteins of the chromosomal passenger complex Aurora B, survivin, and INCENP. In addition to a role in mediating interactions between protein components in the centromere/kinetochore complex, it has also been evidenced that Minor satellite RNA controls the enzymatic function of the Aurora A kinase (Ferri et al. 2009). In addition to centromeric satDNA transcripts, transcripts derived from retrotransposons were also shown to be essential components of the centromere core. For example, in maize, single-stranded non-siRNAs (40-200 nt) transcribed from centromeric CentC satDNA and CRM retrotransposon are tightly bound to CENH3 (Topp et al. 2004). Similarly, RNA transcripts of the LINE-1 retrotransposon were found to bind CENP-A chromatin in Mardel (10) 10q25 neocentromere (Chueh et al. 2009). RNAi-mediated knockdown of the LINE transcripts led to a significant reduction in the mitotic stability of the neocentromere suggesting that retrotransposable elements are a critical epigenetic determinant of the neocentromere. A novel class of small RNAs encompassing contiguous satellites and retroviruses located at the centromere core and likely produced through the activity of retroviral LTR promoters was discovered in a marsupial (Carone et al. 2009). In-depth analysis discovered that hypermorphic expression of these retroelement-encoded small RNAs is critical for the maintenance and assembly of CENP-A in the marsupial centromere (Carone et al. 2013).

\section{Conclusions}

Although being essential for the proper distribution of genetic material in eukaryotic cells, the centromere still continues to intrigue in the complexity of its structure and rapid evolution of its building components. Advances in methodological approaches and high-throughput analyses in the last two decades fostered the rapid accumulation of centromere-related datasets in different model organisms, giving access to information about DNA, RNA, proteins, and their epigenetic modifications. However, the complex networks of interactions among them as well as the details of functional features and roles of particular components are still far from being well understood. Epigenetic determinants are recognized as major identifiers of centromeres in higher eukaryotes, while the functional contribution of DNA remains obscure and seriously questioned because of the ability of the centromere to be formed and to persist on extremely diverse sequences. Recent studies of genomic and functional datasets based on combined sequencing data and established CENH3-associated DNA sequences revealed a more detailed insight into genomic architecture of centromeres. In spite of the diversity of DNA sequences, the preferred forms populating functional centromeres appear to be tandem repetitions of satDNAs and/or mobile elements. Only a subset of centromere-located DNA sequences or their variants is predominantly $\mathrm{CENH} 3$-associated, indicating the importance of their linear composition. An increasing number of reports that evidence organisms with dually organized centromeres (repeat-rich and repeat-free) opens up the possibility that the dynamics of centromere formation is much higher than previously thought, and also highlights stable functioning of centromeres established on different sequence types within a single organism. It can be hypothesized that the repetitive DNA environment has the potential to preserve stability of the functional centromere, and at the same time, to provide a reservoir of new functional sequences. This creates a platform which allows rapid changes in centromere identity and as a consequence can directly stimulate reproductive isolation. Several reasons for this continuous rapid change can be considered, such as specificities of evolution of satDNAs, targeted integration of TEs into the epigenetically marked centromeric environment, and coevolution of DNA 
sequences and CENH3 proteins. The complexity of the DNA sequence and functional relationships in centromeres becomes even more perplexing as a growing number of recent reports indicate roles for centromere DNA transcripts in centromere structure and function. Recent efforts have begun to decipher the rules in sequential patterns of centromeric DNA sequences and their functional interactions in different centromere types which will ultimately lead to a novel integrated view on the centromere genomics.

Acknowledgments We thank Mary Sopta for critical reading of the manuscript. This work was supported by Research Fund of Republic of Croatia, project no. 098-0982913-2756 and by funds received from the European Union's Seventh Framework Programme for research, technological development and demonstration under grant agreement No 316289 - InnoMol, FP7-REGPOT-2012-2013-1.

Open Access This article is distributed under the terms of the Creative Commons Attribution License which permits any use, distribution, and reproduction in any medium, provided the original author(s) and the source are credited.

\section{References}

Alkan C, Cardone MF, Catacchio CR et al (2011) Genome-wide characterization of centromeric satellites from multiple mammalian genomes. Genome Res 21:137-145

Alonso A, Hasson D, Cheung F, Warburton PE (2010) A paucity of heterochromatin at functional human neocentromeres. Epigenetics Chromatin 8:6

Bachmann L, Raab M, Sperlich D (1989) Satellite DNA and speciationa species-specific satellite DNA of Drosophila guanche. J Zool Syst Evol Res 27:84-93

Black BE, Bassett EA (2008) The histone variant CENP-A and centromere specification. Curr Opin Cell Biol 20:91-100

Blower MD, Sullivan BA, Karpen GH (2002) Conserved organization of centromeric chromatin in flies and humans. Dev Cell 2:319-330

Buchwitz BJ, Ahmad K, Moore LL, Roth MB, Henikoff S (1999) A histone-H3-like protein in C. elegans. Nature 401:547-548

Burrack LS, Berman J (2012) Neocentromeres and epigenetically inherited features of centromeres. Chromosome Res 20:607-619

Canapa A, Barucca M, Cerioni PN, Olmo E (2000) A satellite DNA containing CENP-B box-like motifs is present in the antarctic scallop Adamussium colbecki. Gene 247:175-180

Carbone L, Nergadze SG, Magnani E et al (2006) Evolutionary movement of centromeres in horse, donkey, and zebra. Genomics 87:777782

Carone DM, Longo MS, Ferreri GC, Hall L, Harris M, Shook N, Bulazel KV, Carone BR, Obergfell C, O'Neill MJ et al (2009) A new class of retroviral and satellite encoded small RNAs emanates from mammalian centromeres. Chromosoma 118:113-125

Carone DM, Zhang C, Hall LE, Obergfell C, Carone BR, O'Neill MJ, O'Neill RJ (2013) Hypermorphic expression of centromeric retroelement-encoded small RNAs impairs CENP-A loading. Chromosome Res 21:49-62

Chan FL, Wong LH (2012) Transcription in the maintenance of centromere chromatin identity. Nucleic Acids Res 40:11178-11188

Chan FL, Marshall OJ, Saffery R, Kim BW, Earle E, Choo KH, Wong LH (2012) Active transcription and essential role of RNA polymerase II at the centromere during mitosis. Proc Natl Acad Sci U S A 109: 1979-1984
Cheng Z, Dong F, Langdon T, Ouyang S, Buell CR, Gu M, Blattner FR, Jiang J (2002) Functional rice centromeres are marked by a satellite repeat and a centromere-specific retrotransposon. Plant Cell 14: $1691-1704$

Chueh AC, Northrop EL, Brettingham-Moore KH, Choo KH, Wong LH (2009) LINE retrotransposon RNA is an essential structural and functional epigenetic component of a core neocentromeric chromatin. PLoS Genet 5:e1000354

Copenhaver GP, Nickel K, Kuromori T, Benito MI, Kaul S, Lin X, Bevan M, Murphy G, Harris B, Parnell LD, McCombie WR, Martienssen RA, Marra M, Preuss D (1999) Genetic definition and sequence analysis of Arabidopsis centromeres. Science 286:2468-2474

Dawe RK, Henikoff S (2006) Centromeres put epigenetics in the driver's seat. Trends Biochem Sci 31:662-669

De la Herran R, Fontana F, Lanfredi M, Congiu L, Leis M, Rossi R, Ruiz Rejón C, Ruiz Rejón M, Garrido-Ramos MA (2001) Slow rates of evolution and sequence homogenization in an ancient satellite DNA family of sturgeons. Mol Biol Evol 18:432-436

Dernburg AF (2001) Here, there, and everywhere: kinetochore function on holocentric chromosomes. J Cell Biol 153:F33-F38

Dong F, Miller JT, Jackson SA, Wang G-L, Ronald PC, Jiang J (1998) Rice (Oryza sativa) centromeric regions consist of complex DNA. Proc Natl Acad Sci U S A 95:8135-8140

Dover GA (1986) Molecular drive in multigene families: how biological novelties arise, spread and are assimilated. Trends Genet 2:159-165

Durajlija-Žinić S, Ugarković Đ, Cornudella L, Plohl M (2000) A novel interspersed type of organization of satellite DNAs in Tribolium madens heterochromatin. Chromosome Res 8:201-212

Eichler EE (1999) Repetitive conundrums of centromere structure and function. Hum Mol Genet 8:151-155

Ferree PM, Barbash DA (2009) Species-specific heterochromatin prevents mitotic chromosome segregation to cause hybrid lethality in Drosophila. PLoS Biol 7:e1000234

Ferri F, Bouzinba-Segard H, Velasco G, Hubé F, Francastel C (2009) Non-coding murine centromeric transcripts associate with and potentiate Aurora B kinase. Nucleic Acids Res 37:5071-5080

Fishman L, Saunders A (2008) Centromere-associated female meiotic drive entails male fitness costs in monkeyflowers. Science 322:1559-1562

Fry K, Salser W (1977) Nucleotide sequences of HS- alpha satellite DNA from kangaroo rat Dipodomys ordii and characterization of similar sequences in other rodents. Cell 12:1069-1084

Fukagawa T, Nogami M, Yoshikawa M, Ikeno M, Okazaki T, Takami Y, Nakayama T, Oshimura M (2004) Dicer is essential for formation of the heterochromatin structure in vertebrate cells. Nat Cell Biol 6:784-791

Gaffney PM, Pierce JC, Mackinley AG, Titchen DA, Glenn WK (2003) Pearl, a novel family of putative transposable elements in bivalve mollusks. J Mol Evol 56:308-316

Gassmann R, Rechtsteiner A, Yuen KW et al (2012) An inverse relationship to germline transcription defines centromeric chromatin in C. elegans. Nature 484:534-537

Gent JI, Dawe RK (2012) RNA as a structural and regulatory component of the centromere. Annu Rev Genet 46:443-453

Gindullis F, Desel C, Galasso I, Schmidt T (2001) The large-scale organization of the centromeric region in Beta species. Genome Res 11:253-265

Gong Z, Wu Y, Koblízková A et al (2012) Repeatless and repeat-based centromeres in potato: implications for centromere evolution. Plant Cell 24:3559-3574

Grishok A, Tabara H, Mello CC (2000) Genetic requirements for inheritance of RNAi in C. elegans. Science 287:2494-2497

Guenatri M, Bailly D, Maison C, Almouzni G (2004) Mouse centric and pericentric satellite repeats form distinct functional heterochromatin. J Cell Biol 166:493-505

Haizel T, Lim YK, Leitch AR, Moore G (2005) Molecular analysis of holocentric centromeres of Luzula species. Cytogenet Genome Res 109:134-143 
Hall IM, Noma K, Grewal SI (2003) RNA interference machinery regulates chromosome dynamics during mitosis and meiosis in fission yeast. Proc Natl Acad Sci U S A 100:193-198

Han F, Lamb JC, Birchler JA (2006) High frequency of centromere inactivation resulting in stable dicentric chromosomes of maize. Proc Natl Acad Sci U S A 103:3238-3243

Han Y, Zhang Z, Liu C, Liu J, Huang S, Jiang J, Jin W (2009) Centromere repositioning in cucurbit species: implication of the genomic impact from centromere activation and inactivation. Proc Natl Acad Sci U S A 106:14937-14941

Hasson D, Alonso A, Cheung F, Tepperberg JH, Papenhausen PR, Engelen JJ, Warburton PE (2011) Formation of novel CENP-A domains on tandem repetitive DNA and across chromosome breakpoints on human chromosome 8q21 neocentromeres. Chromosoma 120:621-632

Hayden KE, Willard HF (2012) Composition and organization of active centromere sequences in complex genomes. BMC Genomics 13:324

Hayden KE, Strome ED, Merrett SL, Lee HR, Rudd MK, Willard HF (2013) Sequences associated with centromere competency in the human genome. Mol Cell Biol 33:763-772

Heckmann S, Schroeder-Reiter E, Kumke K, Ma L, Nagaki K, Murata M, Wanner G, Houben A (2011) Holocentric chromosomes of Luzula elegans are characterized by a longitudinal centromere groove, chromosome bending, and a terminal nucleolus organizer region. Cytogenet Genome Res 134:220-222

Heckmann S, Macas J, Kumke K, Fuchs J, Schubert V, Ma L, Novák P, Neumann P, Taudien S, Platzer M, Houben A (2013) The holocentric species Luzula elegans shows interplay between centromere and large-scale genome organization. Plant J 73:555-565

Henikoff S, Ahmad K, Malik HS (2001) The centromere paradox: Stable inheritance with rapidly evolving DNA. Science 293:1098-1102

Hillier LW, Miller RD, Baird SE, Chinwalla A, Fulton LA, Koboldt DC, Waterston RH (2007) Comparison of $C$. elegans and C. briggsae genome sequences reveals extensive conservation of chromosome organization and synteny. PLoS Biol 5:e167

Horvath JE, Gulden CL, Vallente RU, Eichler MY, Ventura M, McPherson JD, Graves TA, Wilson RK, Schwartz S, Rocchi M, Eichler EE (2005) Punctuated duplication seeding events during the evolution of human chromosome 2p11. Genome Res 15:914-927

Hosouchi T, Kumekawa N, Tsuruoka H, Kotani H (2002) Physical mapbased sizes of the centromeric regions of Arabidopsis thaliana chromosomes 1, 2, and 3. DNA Res 9:117-121

Hyman AA, Sorger PK (1995) Structure and function of kinetochores in budding yeast. Annu Rev Cell Dev Biol 11:471-495

Jehan Z, Vallinayagam S, Tiwari S, Pradhan S, Singh L, Suresh A, Reddy HM, Ahuja YR, Jesudasan RA (2007) Novel noncoding RNA from human Y distal heterochromatic block (Yq12) generates testisspecific chimeric CDC2L2. Genome Res 17:433-440

Jolly C, Metz A, Govin J, Vigneron M, Turner BM, Khochbin S, Vourc'h C (2004) Stress-induced transcription of satellite III repeats. J Cell Biol 164:25-33

Kalitsis P, Choo KH (2012) The evolutionary life cycle of the resilient centromere. Chromosoma 121:327-340

Kanellopoulou C, Muljo SA, Kung AL, Ganesan S, Drapkin R, Jenuwein T, Livingston DM, Rajewsky K (2005) Dicer-deficient mouse embryonic stem cells are defective in differentiation and centromeric silencing. Genes Dev 19:489-501

Kapitonov VV, Holmquist GP, Jurka J (1998) L1 repeat is a basic unit of heterochromatin satellites in cetaceans. Mol Biol Evol 15:611-612

Kasai F, Garcia C, Arruga MV, Ferguson-Smith MA (2003) Chromosome homology between chicken (Gallus gallus domesticus) and the redlegged partridge (Alectoris rufa); evidence of the occurrence of a neocentromere during evolution. Cytogenet Genome Res 102:326-330

Kato A, Lamb JC, Birchler JA (2004) Chromosome painting using repetitive DNA sequences as probes for somatic chromosome identification in maize. Proc Natl Acad Sci U S A 101:13554-13559
Kazazian HH Jr (2004) Mobile elements: Drivers of genome evolution. Science 303:1626-1632

Kipling D, Warburton PE (1997) Centromeres, CENP-B and Tigger too. Trends Genet 13:141-145

Koo DH, Han F, Birchler JA, Jiang J (2011) Distinct DNA methylation patterns associated with active and inactive centromeres of the maize B chromosome. Genome Res 21:908-914

Kumekawa N, Hosuchi T, Tsuruoka H, Kotani H (2000) The size and sequence organization of the centromeric region of Arabidopsis thaliana chromosome 5. DNA Res 7:315-321

Kuznetsova I, Podgornaya O, Ferguson-Smith MA (2006) Highresolution organization of mouse centromeric and pericentromeric DNA. Cytogenet Genome Res 112:248-255

Lam AL, Boivin CD, Bonney CF, Rudd MK, Sullivan BA (2006) Human centromeric chromatin is a dynamic chromosomal domain that can spread over noncentromeric DNA. Proc Natl Acad Sci U S A 103: 4186-4191

Lamb JC, Meyer JM, Birchler JA (2007) A hemicentric inversion in the maize line knobless Tama flint created two sites of centromeric elements and moved the kinetochore-forming region. Chromosoma 116:237-247

Lee HR, Zhang W, Langdon T, Jin W, Yan H, Cheng Z, Jiang J (2005) Chromatin immunoprecipitation cloning reveals rapid evolutionary patterns of centromeric DNA in Oryza species. Proc Natl Acad Sci U S A 102:11793-11798

Li B, Choulet F, Heng Y, Hao W, Paux E, Liu Z, Yue W, Jin W, Feuillet C, Zhang X (2013) Wheat centromeric retrotransposons: the new ones take a major role in centromeric structure. Plant J 73:952-965

Ma J, Jackson SA (2006) Retrotransposon accumulation and satellite amplification mediated by segmental duplication facilitate centromere expansion in rice. Genome Res 16:251-259

Ma J, Wing RA, Bennetzen JL, Jackson SA (2007) Plant centromere organization: a dynamic structure with conserved functions. Trends Genet 23:134-139

Macas J, Koblízková A, Navrátilová A, Neumann P (2009) Hypervariable 3' UTR region of plant LTR-retrotransposons as a source of novel satellite repeats. Gene 448:198-206

Macas J, Neumann P, Novák P, Jiang J (2010) Global sequence characterization of rice centromeric satellite based on oligomer frequency analysis in large-scale sequencing data. Bioinformatics 26:21012108

Mahtani MM, Willard HF (1998) Physical and genetic mapping of the human $\mathrm{X}$ chromosome centromere: repression of recombination. Genome Res 8:100-110

Malik HS, Henikoff S (2001) Adaptive evolution of Cid, a centromerespecific histone in Drosophila. Genetics 157:1293-1298

Malik HS, Henikoff S (2009) Major evolutionary transitions in centromere complexity. Cell 138:1067-1082

Maloney KA, Sullivan LL, Matheny JE, Strome ED, Merrett SL, Ferris A, Sullivan BA (2012) Functional epialleles at an endogenous human centromere. Proc Natl Acad Sci U S A 109:13704-13709

Marshall OJ, Chueh AC, Wong LH, Choo KH (2008) Neocentromeres: new insights into centromere structure, disease development, and karyotype evolution. Am J Hum Genet 82:261-282

Masabanda JS, Burt DW, O'Brien PC et al (2004) Molecular cytogenetic definition of the chicken genome: the first complete avian karyotype. Genetics 166:1367-1373

Masumoto H, Nakano M, Ohzeki J (2004) The role of CENP-B and alpha-satellite DNA: de novo assembly and epigenetic maintenance of human centromeres. Chromosome Res 12:543-556

May BP, Lippman ZB, Fang Y, Spector DL, Martienssen RA (2005) Differential regulation of strand-specific transcripts from Arabidopsis centromeric satellite repeats. PLoS Genet 1:e79

Melters DP, Bradnam KR, Young HA et al (2013) Comparative analysis of tandem repeats from hundreds of species reveals unique insights into centromere evolution. Genome Biol 14:R10 
Meštrović N, Plohl M, Mravinac B, Ugarković Đ (1998) Evolution of satellite DNAs from the genus Palorus - Experimental evidence for the "library" hypothesis. Mol Biol Evol 15:1062-1068

Meštrović N, Pavlek M, Car A, Castagnone-Sereno P, Abad P, Plohl M (2013) Conserved DNA motifs, including the CENP-B box-like, are possible promoters of satellite DNA array rearrangements in nematodes. PLoS One 8:e6732

Mola LM, Papeschi AG (2006) Holokinetic chromosomes at a glance. BAG J Basic Appl Genet 17:17-33

Mravinac B, Plohl M (2010) Parallelism in evolution of highly repetitive DNAs in sibling species. Mol Biol Evol 27:1857-1867

Mravinac B, Plohl M, Meštrović N, Ugarković Đ (2002) Sequence of PRAT satellite DNA "frozen" in some Coleopteran species. J Mol Evol 54:774-783

Mravinac B, Ugarković Đ, Franjević D, Plohl M (2005) Long inversely oriented subunits form a complex monomer of Tribolium brevicornis satellite DNA. J Mol Evol 60:513-525

Mravinac B, Sullivan LL, Reeves JW, Yan CM, Kopf KS, Farr CJ, Schueler MG, Sullivan BA (2009) Histone modifications within the human X centromere region. PLoS One 4:e6602

Murchison EP, Partridge JF, Tam OH, Cheloufi S, Hannon GJ (2005) Characterization of Dicer-deficient murine embryonic stem cells. Proc Natl Acad Sci U S A 102:12135-12140

Nagaki K, Talbert PB, Zhong CX, Dawe RK, Henikoff S, Jiang J (2003) Chromatin immunoprecipitation reveals that the 180-bp satellite repeat is the key functional DNA element of Arabidopsis thaliana centromeres. Genetics 163:1221-1225

Nagaki K, Cheng Z, Ouyang S, Talbert PB, Kim M, Jones KM, Henikoff S, Buell CR, Jiang J (2004) Sequencing of a rice centromere uncovers active genes. Nat Genet 36:138-145

Nagaki K, Kashihara K, Murata M (2005) Visualization of diffuse centromeres with centromere-specific histone $\mathrm{H} 3$ in the holocentric plant Luzula nivea. Plant Cell 17:1886-1893

Neumann P, Navrátilová A, Koblížková A, Kejnovský E, Hřibová E, Hobza R, Widmer A, Doležel J, Macas J (2011) Plant centromeric retrotransposons: a structural and cytogenetic perspective. Mob DNA 2:4

Neumann P, Navrátilová A, Schroeder-Reiter E, Koblížková A, Steinbauerová V, Chocholová E, Novák P, Wanner G, Macas J (2012) Stretching the rules: monocentric chromosomes with multiple centromere domains. PLoS Genet 8:e1002777

Ohta T, Dover GA (1984) The cohesive population genetics of molecular drive. Genetics 108:501-521

Ohzeki J, Nakano M, Okada T, Masumoto H (2002) CENP-B box is required for de novo centromere chromatin assembly on human alphoid DNA. J Cell Biol 159:765-775

Okamoto Y, Nakano M, Ohzeki J, Larionov V, Masumoto H (2007) A minimal CENP-A core is required for nucleation and maintenance of a functional human centromere. EMBO J 26:1279-1291

Pal-Bhadra M, Leibovitch BA, Gandhi SG, Rao M, Bhadra U, Birchler JA, Elgin SC (2004) Heterochromatic silencing and HP1 localization in Drosophila are dependent on the RNAi machinery. Science 303:669-672

Palomeque T, Lorite P (2008) Satellite DNA in insects: a review. Heredity 100:564-573

Pimpinelli S, Berloco M, Fanti L, Dimitri P, Bonaccorsi S, Marchetti E, Caizzi R, Caggese C, Gatti M (1995) Transposable elements are stable structural components of Drosophila melanogaster heterochromatin. Proc Natl Acad Sci U S A 92:3804-3808

Piras FM, Nergadze SG, Magnani E, Bertoni L, Attolini C, Khoriauli L, Raimondi E, Giulotto E (2010) Uncoupling of satellite DNA and centromeric function in the genus Equus. PLoS Genet 6:e1000845

Plohl M, Luchetti A, Meštrović N, Mantovani B (2008) Satellite DNAs between selfishness and functionality: structure, genomics and evolution of tandem repeats in centromeric (hetero)chromatin. Gene 409:72-82
Plohl M, Meštrović N, Mravinac B (2012) Satellite DNA evolution. In: Garrido-Ramos MA (ed) Repetitive DNA, Genome dynamics, 7th edn. Karger, Basel, pp 126-152

Pluta AF, Mackay AM, Ainsztein AM, Goldberg IG, Earnshaw WC (1995) The centromere: hub of chromosomal activities. Science 270:1591-1594

Probst AV, Okamoto I, Casanova M, El Marjou F, Le Baccon P, Almouzni G (2010) A strand-specific burst in transcription of pericentric satellites is required for chromocenter formation and early mouse development. Dev Cell 19:625-638

Reyes-Turcu FE, Zhang K, Zofall M, Chen E, Grewal SIS (2011) Defects in RNA quality control factors reveal RNAi-independent nucleation of heterochromatin. Nat Struct Mol Biol 18:1132-1138

Rocchi M, Archidiacono N, Schempp W, Capozzi O, Stanyon R (2012) Centromere repositioning in mammals. Heredity 108:59-67

Rudd MK, Willard H (2004) Analysis of the centromeric regions of the human genome assembly. Trends Genet 20:529-533

Rudd MK, Wray GA, Willard HF (2006) The evolutionary dynamics of alpha-satellite. Genome Res 16:88-96

Sato H, Masuda F, Takayama Y, Takahashi K, Saitoh S (2012) Epigenetic inactivation and subsequent heterochromatinization of a centromere stabilize dicentric chromosomes. Curr Biol 22:658-667

Schueler MG, Higgins AW, Rudd MK, Gustashaw K, Willard HF (2001) Genomic and genetic definition of a functional human centromere. Science 294:109-115

Shang WH, Hori T, Toyoda A, Kato J, Popendorf K, Sakakibara Y, Fujiyama A, Fukagawa T (2010) Chickens possess centromeres with both extended tandem repeats and short non-tandemrepetitive sequences. Genome Res 20:1219-1228

Sharma A, Wolfgruber TK, Presting GG (2013) Tandem repeats derived from centromeric retrotransposons. BMC Genomics 14:142

Smith GP (1976) Evolution of repeated DNA sequences by unequal crossover. Science 191:528-535

Smith CD, Shu S, Mungall CJ, Karpen GH (2007) The Release 5.1 annotation of Drosophila melanogaster heterochromatin. Science 316:1586-1591

Spence JM, Critcher R, Ebersole TA, Valdivia MM, Earnshaw WC, Fukagawa T, Farr CJ (2002) Co-localization of centromere activity, proteins and topoisomerase II within a subdomain of the major human X alpha-satellite array. EMBO J 21:5269-5280

Stimpson KM, Song IY, Jauch A, Holtgreve-Grez H, Hayden KE, Bridger JM, Sullivan BA (2010) Telomere disruption results in non-random formation of de novo dicentric chromosomes involving acrocentric human chromosomes. PLoS Genet 6:e1001061

Strachan T, Webb D, Dover GA (1985) Transition stages of molecular drive in multiple-copy DNA families in Drosophila. EMBO J 4: 1701-1708

Sullivan BA, Willard HF (1998) Stable dicentric X chromosomes with two functional centromeres. Nat Genet 20:227-228

Sullivan BA, Karpen GH (2004) Centromeric chromatin exhibits a histone modification pattern that is distinct from both euchromatin and heterochromatin. Nat Struct Mol Biol 11:10761083

Sullivan LL, Boivin CD, Mravinac B, Song IY, Sullivan BA (2011) Genomic size of CENP-A domain is proportional to total alpha satellite array size at human centromeres and expands in cancer cells. Chromosome Res 19:457-470

Sun X, Wahlstrom J, Karpen G (1997) Molecular structure of a functional Drosophila centromere. Cell 91:1007-1019

Sun X, Le HD, Wahlstrom JM, Karpen GH (2003) Sequence analysis of a functional Drosophila centromere. Genome Res 13:182-194

Talbert PB, Henikoff S (2010) Centromeres convert but don't cross. PLoS Biol 8:e1000326

Talbert PB, Masuelli R, Tyagi AP, Comai L, Henikoff S (2002) Centromeric localization and adaptive evolution of an Arabidopsis histone H3 variant. Plant Cell 14:1053-1066 
Talbert PB, Bryson TD, Henikoff S (2004) Adaptive evolution of centromere proteins in plants and animals. J Biol 3:18

Thompson SL, Bakhoum SF, Compton DA (2010) Mechanisms of chromosomal instability. Curr Biol 20:R285-95

Ting DT, Lipson D, Paul S et al (2011) Aberrant overexpression of satellite repeats in pancreatic and other epithelial cancers. Science 331:593-596

Tollis M, Boissinot S (2012) The evolutionary dynamics of transposable elements in eukaryote genomes. In: Garrido-Ramos MA (ed) Repetitive DNA, Genome dynamics, 7th edn. Karger, Basel, pp 68-91

Topp CN, Zhong CX, Dawe RK (2004) Centromere-encoded RNAs are integral components of the maize kinetochore. Proc Natl Acad Sci U S A 101:15986-15991

Tsukahara S, Kawabe A, Kobayashi A, Ito T, Aizu T, Shin-i T, Toyoda A, Fujiyama A, Tarutani Y, Kakutani T (2012) Centromere-targeted de novo integrations of an LTR retrotransposon of Arabidopsis lyrata. Genes Dev 26:705-713

Ventura M, Antonacci F, Cardone MF, Stanyon R, D'Addabbo P, Cellamare A, Sprague LJ, Eichler EE, Archidiacono N, Rocchi M (2007) Evolutionary formation of new centromeres in macaque. Science 316:243-246

Volpe TA, Kidner C, Hall IM, Teng G, Grewal SI, Martienssen RA (2002) Regulation of heterochromatic silencing and histone H3 lysine- 9 methylation by RNAi. Science 297:1833-1837

Vourc'h C, Biamonti G (2011) Transcription of Satellite DNAs in Mammals. Prog Mol Subcell Biol 51:95-118

Waye JS, Willard HF (1989) Human beta satellite DNA: genomic organization and sequence definition of a class of highly repetitive tandem DNA. Proc Natl Acad Sci U S A 86: $6250-6254$

Willard HF, Waye JS (1987) Chromosome-specific subsets of human alpha satellite DNA: analysis of sequence divergence within and between chromosomal subsets and evidence for an ancestral pentameric repeat. J Mol Evol 25:207-214

Wolfgruber TK, Sharma A, Schneider KL et al (2009) Maize centromere structure and evolution: sequence analysis of centromeres 2 and 5 reveals dynamic Loci shaped primarily by retrotransposons. PLoS Genet 5:e1000743

Wong LH, Brettingham-Moore KH, Chan L et al (2007) Centromere RNA is a key component for the assembly of nucleoproteins at the nucleolus and centromere. Genome Res 17:1146-1160

Wu J, Yamagata H, Hayashi-Tsugane M et al (2004) Composition and structure of the centromeric region of rice chromosome 8. Plant Cell 16:967-976

Zhang W, Friebe B, Gill BS, Jiang J (2010) Centromere inactivation and epigenetic modifications of a plant chromosome with three functional centromeres. Chromosoma 119:553-563

Zhong CX, Marshall JB, Topp C, Mroczek R, Kato A, Nagaki K, Birchler JA, Jiang JM, Dawe RK (2002) Centromeric retroelements and satellites interact with maize kinetochore protein $\mathrm{CENH}$. Plant Cell 14:2825-2836

Zhu Q, Pao GM, Huynh AM, Suh H, Tonnu N, Nederlof PM, Gage FH, Verma IM (2011) BRCA1 tumour suppression occurs via heterochromatin-mediated silencing. Nature 477:179-184

Zilberman D, Cao X, Jacobsen SE (2003) ARGONAUTE4 control of locus-specific siRNA accumulation and DNA and histone methylation. Science 299:716-719 